\title{
Bi-Cyclide and Flat-Ring Cyclide Coordinate Surfaces: Correction of Two Expressions
}

\author{
By Philip W. Kuchel, Brian T. Bulliman, and Edward D. Fackerell
}

\begin{abstract}
Bi-cyclide and flat-ring cyclide coordinates are three-dimensional rotational coordinate systems based on conformal transformations using the Jacobian elliptic function sn. We have checked the previously published formulae of these systems (P. Moon and D. E. Spencer. Field Theory Handhook. Springer-Verlag. Berlin. 1971). In both cases the expression for the rotation-cyclide surfaces was incorrect: thus we present rederivations. The expressions were verified with the symbolic-algebraic computation package MACSYMA.
\end{abstract}

1. Introduction. Novel orthogonal coordinate systems in two dimensions can be generated by conformal transformations using analytic functions of complex variables; three-dimensional systems follow by rotation about either the real or the imaginary axes [8], [9]. Our interest in these systems is related to the calculation of the magnetic potential in and around nonspherical objects introduced into a uniform magnetic field; of particular interest are the biconcave-disc shapes of some red blood cells [3]. Among the analytic functions that yield coordinate curves that are similar to the cross section of biconcave discs is the Jacobian elliptic function $z=x+i y=$ $a \operatorname{sn}(w, k)$ [6]. [7], where $a$ is real and the complex numbers $w=\mu+i \nu$ and $k$ are the argument and modulus, respectively [1]. Separation of the real and imaginary parts of the elliptic function yields two coordinate-transformation equations, in $x$ and $y[6]-[9]$ :

$$
\begin{aligned}
& x=\frac{a}{\Lambda} \operatorname{sn} \mu \operatorname{dn} \nu^{\prime} . \\
& y=\frac{a}{\Lambda} \operatorname{cn} \mu \operatorname{dn} \mu \operatorname{sn} \nu^{\prime} \operatorname{cn} \nu^{\prime}, \\
& \Lambda=1-\operatorname{dn}^{2} \mu \operatorname{sn}^{2} \nu^{\prime}, \\
& 0 \leqslant \mu \leqslant K, \quad 0 \leqslant \nu \leqslant K^{\prime} .
\end{aligned}
$$

where $K$ and $K^{\prime}$ are the definite elliptic integrals of the first kind with respect to $k$ and its complement $k^{\prime}$, respectively [2], [5]; and the prime on $\nu^{\prime}$ specifies that $k^{\prime}$ is used in the elliptic function. The series of coordinate curves shown in Figure 1.1 were drawn for three different values of $k$ in order to emphasize the effects of changes in $k$ on the concavity of the $\nu=$ constant curves. Moon and Spencer have already presented similar curves, but only for $k^{2}=0.5[6]-[9]$.

Received December 2. 1985: revised March 5. 1986. October 20. 1986. and January 28. 1987. 1980 Mathematics Subject Classification (1985 Rerision). Primary 33A25. 

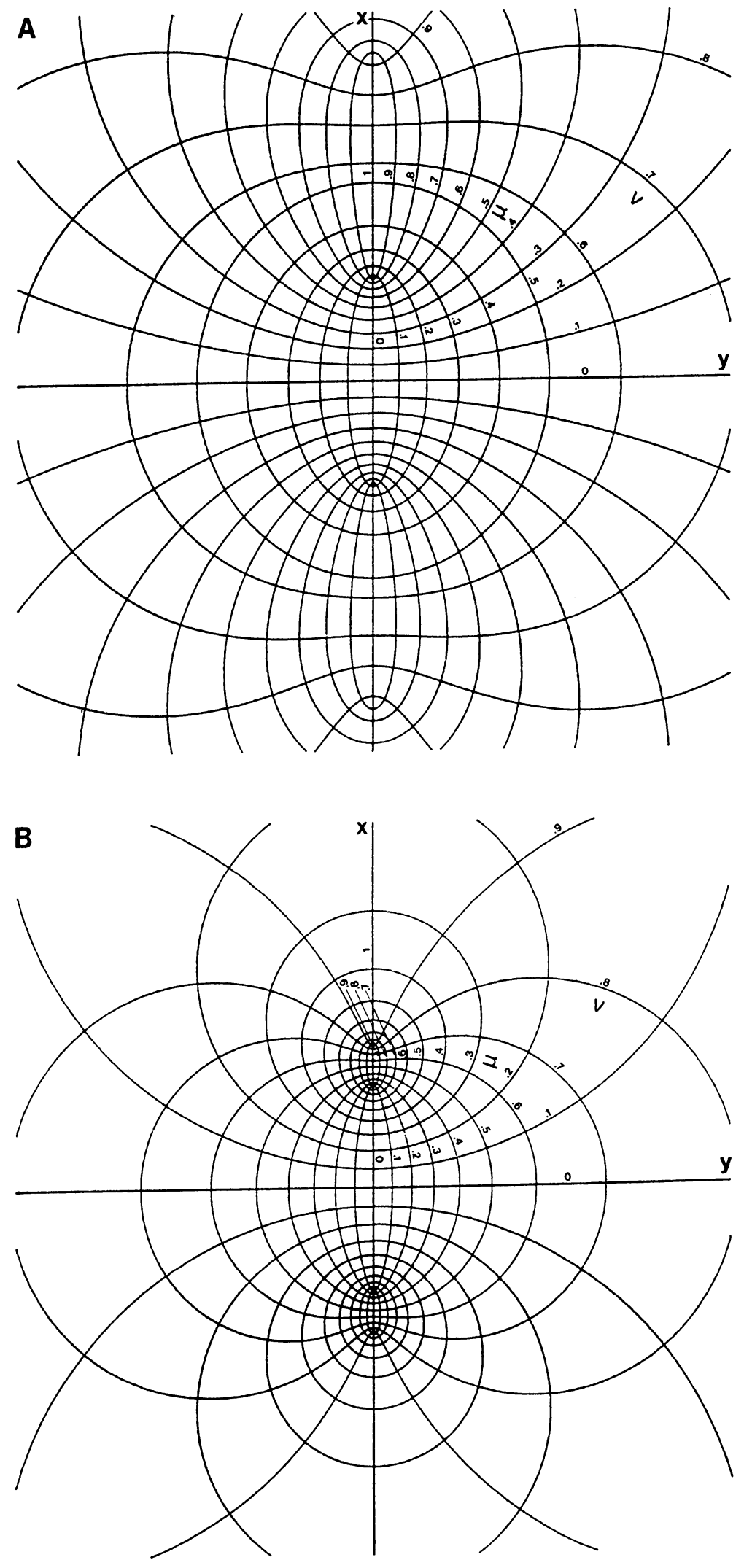


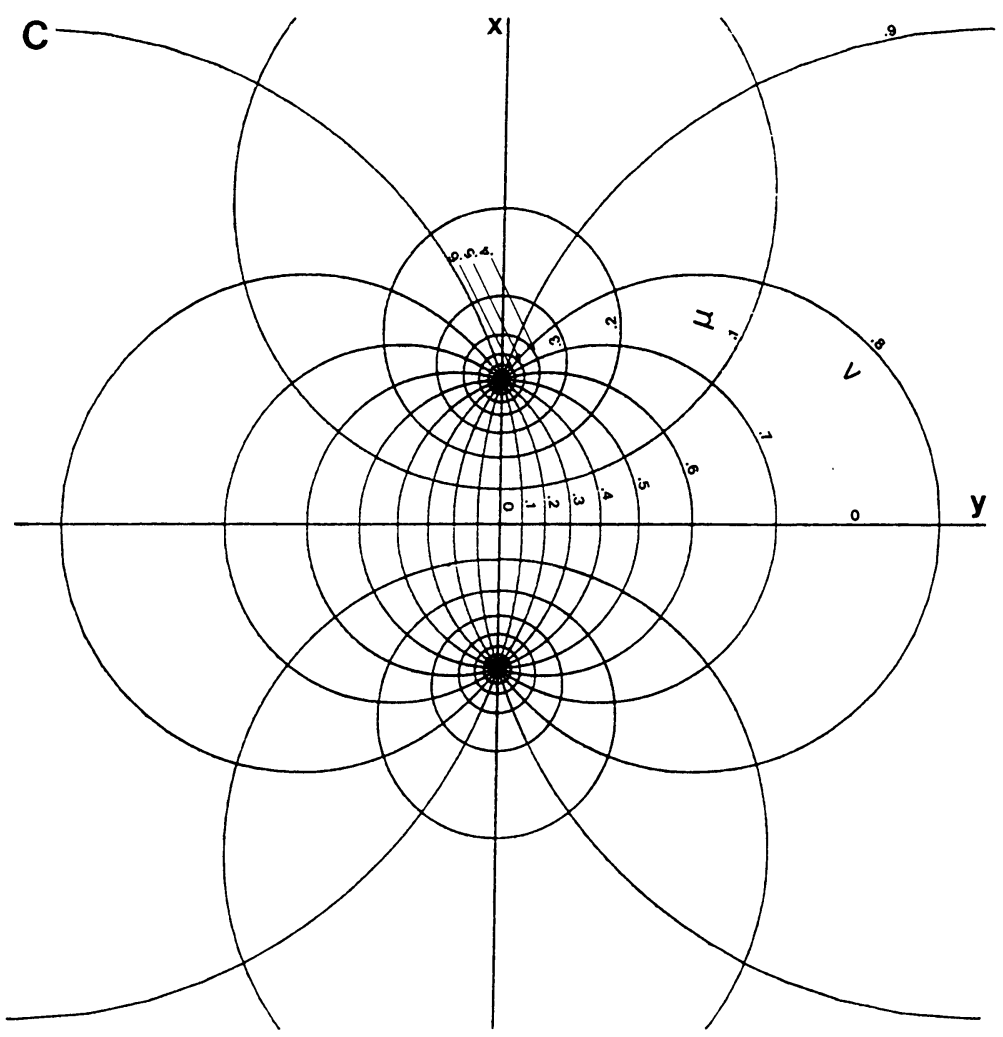

FIGURE 1.1

Orthogonal closed-curve coordinate system described by (1.1)-(1.4). The following values of real $a$ and $k^{2}$ were used: A, 30, 0.1; B, 30, 0.5; C, 30, 0.9. $K$ and $K^{\prime}$ were calculated by computer using the hypergeometric series expression $[2$, p. 298] and sn was evaluated using the series expression [5, p. 13] programmed in BASIC. The scale-values of $\mu$ and $\nu$ are fractions of $K$ and $K^{\prime}$, respectively. The curves were plotted using a Hewlett-Packard Series 9000 model 220 computer and a $7475 \mathrm{~A}$ plotter.

When the maps of Figure 1.1 are rotated about the $x$-axis, we obtain an orthogonal family of surfaces [6], [7]. The Cartesian transformations are given in terms of the bi-cyclide coordinates $(\mu, \nu, \psi)$;

$$
\begin{aligned}
& x=\frac{a}{\Lambda} \operatorname{cn} \mu \operatorname{dn} \mu \operatorname{sn} \nu^{\prime} \operatorname{cn} \nu^{\prime} \cos \psi, \\
& y=\frac{a}{\Lambda} \operatorname{cn} \mu \operatorname{dn} \mu \operatorname{sn} \nu^{\prime} \operatorname{cn} \nu^{\prime} \sin \psi, \\
& z=\frac{a}{\Lambda} \operatorname{sn} \mu \operatorname{dn} \nu^{\prime},
\end{aligned}
$$

Expressions for the three families of coordinate surfaces (bi-cyclides, $\mu=$ constant; rotation cyclides, $\nu=$ constant; meridional half-planes, $\psi=$ constant) are obtained by elimination of two of the three bi-cyclide variables from (1.5) to (1.7). 
2. Derivation of Expressions for Coordinate Surfaces. The process of variableelimination from (1.5) to (1.7) was as follows. Let,

$$
\begin{array}{lll}
s=\operatorname{sn}(\mu, k), & c=\operatorname{cn}(\mu, k), & d=\operatorname{dn}(\mu, k) . \\
S=\operatorname{sn}\left(\nu, k^{\prime}\right), & C=\operatorname{cn}\left(\nu, k^{\prime}\right), & D=\operatorname{dn}\left(\nu, k^{\prime}\right), \quad k^{\prime 2}=1-k^{2}, \\
r^{2}=x^{2}+y^{2}+z^{2} . &
\end{array}
$$

2.1. $\nu=$ constant. The Cartesian coordinate surface for this condition was derived by eliminating $\psi$ and $\mu$ from (1.5) and (1.6); $\psi$ was eliminated by squaring these equations followed by addition and using $\cos ^{2} \psi+\sin ^{2} \psi=1$. Thus, from (1.3),

$$
\Lambda=1-d^{2} S^{2}=C^{2}+k^{2} S^{2} s^{2}
$$

from (1.7),

$$
\Lambda z / a=s D
$$

and from (1.5) to (1.7),

$$
\Lambda^{2}\left(r^{2}-z^{2}\right) / a^{2}=c^{2} d^{2} S^{2} C^{2} .
$$

Squaring both sides of (2.2) and using $D^{2}=1-k^{\prime 2} S^{2}=C^{2}+k^{2} S^{2}$ gives

$$
\Lambda^{2} z^{2} / a^{2}=s^{2}\left(C^{2}+k^{2} S^{2}\right)=\left(1-c^{2}\right) C^{2}+\left(1-d^{2}\right) S^{2}=1-c^{2} C^{2}-d^{2} S^{2} .
$$

Adding this to (2.3) gives

$$
\begin{aligned}
& \Lambda^{2} r^{2} / a^{2}=\left(1-c^{2} C^{2}\right)\left(1-d^{2} S^{2}\right)=\left(1-c^{2} C^{2}\right) \Lambda . \\
& \Lambda r^{2} / a^{2}=1-c^{2} C^{2}=S^{2}+s^{2} C^{2}=s^{2}+c^{2} S^{2} .
\end{aligned}
$$

We now have

$$
\begin{aligned}
\Lambda^{2} & =\left(C^{2}+k^{2} S^{2} s^{2}\right)^{2}, \\
\Lambda^{2} z^{2} / a^{2} & =D^{2} s^{2}, \\
\Lambda^{2} r^{2} / a^{2} & =S^{2} C^{2}+\left(C^{4}+k^{2} S^{4}\right) s^{2}+k^{2} S^{2} C^{2} s^{4}, \\
\Lambda^{2} r^{4} / a^{4} & =\left(S^{2}+C^{2} s^{2}\right)^{2} .
\end{aligned}
$$

The right-hand sides are four linear combinations of the three quantities $s^{4}, s^{2}$, and $s^{0}$, which can be eliminated to yield a linear homogeneous relation between the four left sides. Using the identity $C^{4}-k^{2} S^{4}=C^{2}-S^{2} D^{2}$ and cancellation of $\Lambda^{2}$ gives

$$
\frac{k^{2} r^{4}}{a^{4}}-\left(\frac{C^{4}+k^{2} S^{4}}{S^{2} C^{2}}\right) \frac{r^{2}}{a^{2}}+\frac{\left(C^{2}-S^{2} D^{2}\right)^{2}}{S^{2} C^{2} D^{2}} \frac{z^{2}}{a^{2}}+1=0 .
$$

To obtain the basic equation-form given by Moon and Spencer [6], [7], [9], we expand the coefficients of (2.9) in sn $\nu^{\prime}$ only:

$$
\left(x^{2}+y^{2}+z^{2}\right)^{2}-P\left(x^{2}+y^{2}\right)-Q z^{2}-R=0
$$


where

$$
\begin{aligned}
P & =\frac{a^{2}}{k^{2}}\left[\frac{\left(1+k^{2}\right) \mathrm{sn}^{4} \nu^{\prime}-2 \mathrm{sn}^{2} \nu^{\prime}+1}{\left(1-\mathrm{sn}^{2} \nu^{\prime}\right) \mathrm{sn}^{2} \nu^{\prime}}\right], \\
Q & =P-\frac{\left(C^{2}-S^{2} D^{2}\right)^{2}}{S^{2} C^{2} D^{2}} \frac{a^{2}}{k^{2}}=\frac{a^{2}}{k^{2}}\left[\frac{S^{2} C^{2} D^{4}+k^{2} S^{2} C^{2}}{S^{2} C^{2} D^{2}}\right] \\
& =\frac{a^{2}}{k^{2}}\left[\frac{\left(k^{2}-1\right)^{2} \operatorname{sn}^{4} \nu^{\prime}+2\left(k^{2}-1\right) \operatorname{sn}^{2} \nu^{\prime}+\left(k^{2}+1\right)}{\left(k^{2}-1\right) \mathrm{sn}^{2} \nu^{\prime}+1}\right], \\
R & =-\frac{a^{4}}{k^{2}} .
\end{aligned}
$$

Expression (2.10) and its coefficients differs from that given by Moon and Spencer [9, p. 124]. The expressions were, in fact, first derived here using the symbolic-algebraic computation package MACSYMA [4]. Batch-mode procedure files used for computations relating to this and other sections are available from the authors.

2.2. $\mu=$ constant. The right-hand sides of (2.5) to (2.8) can be re-expressed as linear combinations of $S^{4}, S^{2}$, and $S^{0}$ with coefficients depending on the lower-case letters. Elimination of these capitals yielded the coordinate surfaces defined by Eq.

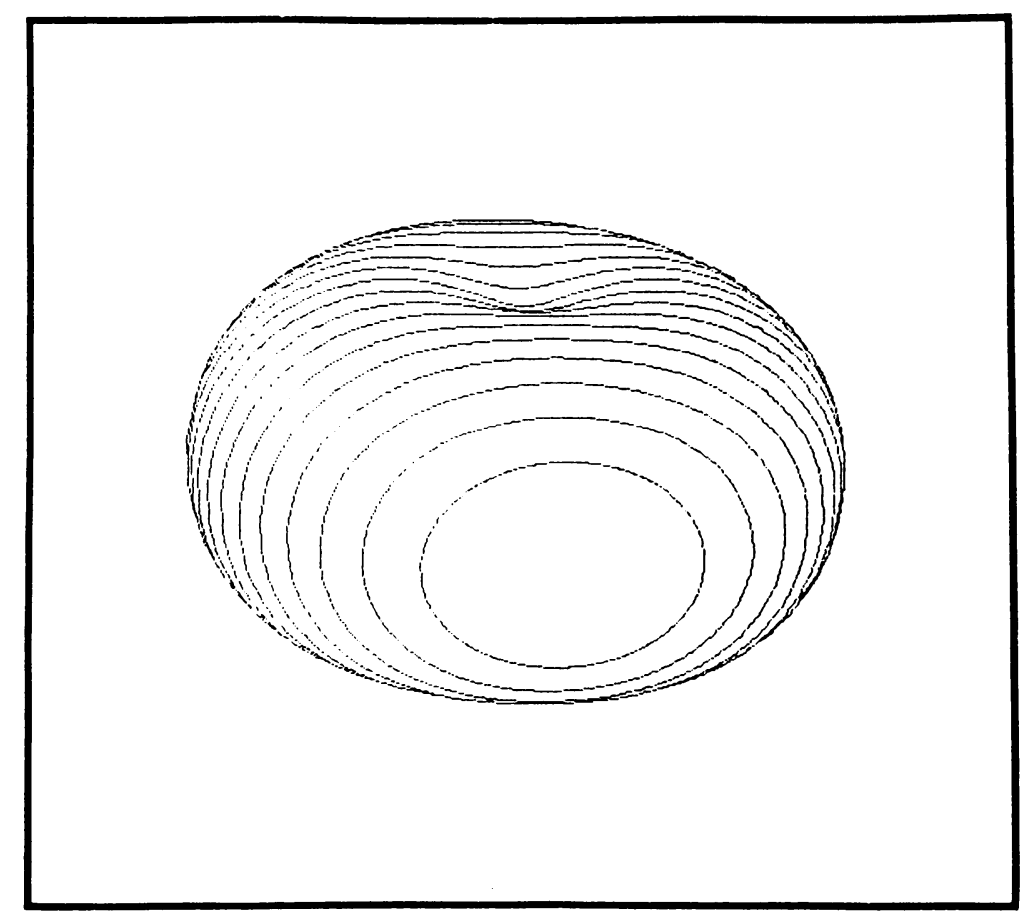

FIGURE 2.1

Two-dimensional projection of a rotation cyclide coordinate surface of the bi-cyclide coordinate system. The parameter values used in Eqs. (2.10) to (2.13) for this computer-based drawing were $a=3.0, k^{2}=0.1, \nu=0.8 K^{\prime}$. The curves were plotted using a Hewlett-Packard Think Jet printer from a screen dump from the computer mentioned in the caption of Figure 1.1 . 
(2.10) with the following coefficients:

$$
\begin{aligned}
& P=-\frac{a^{2}}{k^{2}}\left[\frac{k^{2} \mathrm{cn}^{4} \mu+\mathrm{dn}^{4} \mu}{\mathrm{cn}^{2} \mu \mathrm{dn}^{2} \mu}\right], \\
& Q=\frac{a^{2}}{k^{2}}\left[k^{2} \operatorname{sn}^{2} \mu+\frac{1}{\mathrm{sn}^{2} \mu}\right], \\
& R=-\frac{a^{4}}{k^{2}} .
\end{aligned}
$$

These expressions, after some rearrangement, are the same as Moon and Spencer's $[9$, p. 124].

2.3. $\psi=$ constant. This coordinate surface is simply the half-plane given by $\tan \psi=y / x[9]$.

2.4. Graphical Representation of Surfaces. Figure 2.1 is a two-dimensional projection of a rotation cyclide obtained using computer graphics which relied on the expressions (2.10) to (2.13).

3. Flat-Ring Cyclide Coordinates $(\mu, \nu, \psi)$. The Cartesian transformations for this coordinate system are [7], [8],

$$
\begin{aligned}
& x=\frac{a}{\Lambda} \operatorname{sn} \mu \operatorname{dn} \nu^{\prime} \cos \psi, \\
& y=\frac{a}{\Lambda} \operatorname{sn} \mu \operatorname{dn} \nu^{\prime} \sin \psi, \\
& z=\frac{a}{\Lambda} \operatorname{cn} \mu \operatorname{dn} \mu \operatorname{sn} \nu^{\prime} \operatorname{cn} \nu^{\prime},
\end{aligned}
$$

where $\Lambda$ and the ranges of the variables are as in (1.3) and (1.8).

The equations of the coordinate surfaces were derived in the same way as the bi-cyclide cases, after noting that (2.8) still holds, although the roles of $z^{2}$ and $r^{2}-z^{2}$ are interchanged in the derivation. We confirmed the correctness of Moon and Spencer's expression [8, p. 127] for the flat ring-cyclides ( $\mu$ = constant). However, the formula for the rotation cyclides $(\nu=$ constant $)$ was shown to be wrong. The correct expressions for the coefficients in (2.10) are,

$$
\begin{aligned}
& P=\frac{a^{2}}{k^{2}}\left[\frac{\left(k^{2}-1\right)^{2} \operatorname{sn}^{4} \nu^{\prime}+2\left(k^{2}-1\right) \operatorname{sn}^{2} \nu^{\prime}+\left(k^{2}+1\right)}{\left(k^{2}-1\right) \operatorname{sn}^{2} \nu^{\prime}+1}\right] \equiv(2.12), \\
& Q=\frac{a^{2}}{k^{2}}\left[\frac{\left(1+k^{2}\right) \operatorname{sn}^{4} \nu^{\prime}-2 \operatorname{sn}^{2} \nu^{\prime}+1}{\left(1-\operatorname{sn}^{2} \nu^{\prime}\right) \operatorname{sn}^{2} \nu^{\prime}}\right] \equiv(2.11), \\
& R=-\frac{a^{4}}{k^{2}}
\end{aligned}
$$

4. Discussion. That the earlier versions of the expressions for the coordinate surfaces, $\nu=$ constant, are incorrect can be demonstrated readily by choosing values of $\nu$ and substituting the corresponding values of the relevant elliptic functions into them. Fortuitously, if $k^{2}$ has the value 0.5 (as was used by Moon and Spencer [7], [8]), then, for a wide range of $\nu$ and $a$ values the previous 'equality' is satisfied to within $<0.01 a$. However, if $k^{2} \neq 0.5$, a much larger error can appear with the previously published equations; this is not the case with our expressions. We are 
uncertain whether a systematic error arose in the earlier derivations of the formulae; but we have excluded, by use of MACSYMA [4], the suggestion that $k$ instead of $k^{\prime}$ was used in the expressions containing $\nu$.

Acknowledgments. The financial assistance of the Australian National Health and Medical Research Council and the Australian Research Grants Scheme is gratefully acknowledged. Mr. Adam Hudson is thanked for assistance with MACSYMA.

Department of Biochemistry

University of Sydney

Sydney, NSW 2006, Australia

Department of Applied Mathematics

University of Sydney

Sydney, NSW 2006, Australia

1. F. Bowman, Introduction to Elliptic Functions with Applications, Dover, New York, 1961.

2. P. F. Byrd \& M. D. Friedman, Handbook of Elliptic Integrals for Engineers and Scientists, Springer-Verlag, Berlin, 1971.

3. Z. H. Endre, P. W. Kuchel \& B. E. Chapman, "Cell volume dependence of ${ }^{1} H$ spin-echo NMR signals in human erythrocyte suspensions: The influence of in situ field gradients," Biochim. Biophys. Acta, v. 803, 1984, pp. 137-144.

4. Mathlab Group, Laboratory for Computer SCIENCE, macSYMa Reference Manual, Version 10. Massachusetts Institute of Technology, Cambridge, Mass., 1983.

5. L. M. Milne-Thomson, Jacobian Elliptic Function Tables: A Guide to Practical Computation with Elliptic Functions and Integrals together with Tables of $\operatorname{sn} \mu, \mathrm{cn} \mu, \operatorname{dn} \mu, Z(u)$, Macmillan, London, 1970.

6. P. MOON \& D. E. SPENCER, "Cylindrical and rotational coordinate systems," J. Franklin Inst., v. 252, 1951, pp. 327-344.

7. P. MOON \& D. E. SPencer, "Some coordinate systems associated with elliptic functions," $J$. Franklin Inst., v. 255, 1953, pp. 531-543.

8. P. MOON \& D. E. SPENCER, Field Theory for Engineers, Van Nostrand, Princeton, N. J., 1961.

9. P. MOon \& D. E. SPENCER, Field Theory Handbook Including Coordinate Systems, Differential Equations and Their Solutions, Springer-Verlag, Berlin, 1971. 